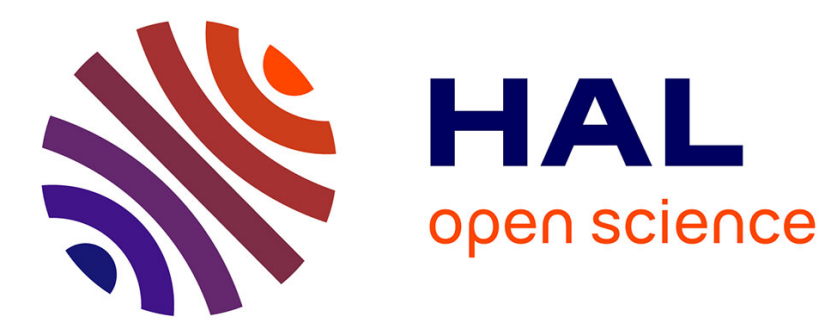

\title{
MESURE DE LA FONCTION DE TRANSFERT ÉLECTRO-OPTIQUE D'UN SYSTĖME ÉLECTROCHIMIQUE
}

\author{
G. Blondeau, M. Froelicher, Claude Gabrielli, Anne Hugot-Le Goff, V. \\ Jovancicevic
}

\section{To cite this version:}

G. Blondeau, M. Froelicher, Claude Gabrielli, Anne Hugot-Le Goff, V. Jovancicevic. MESURE DE LA FONCTION DE TRANSFERT ÉLECTRO-OPTIQUE D'UN SYSTĖME ÉLECTROCHIMIQUE. Journal de Physique Colloques, 1977, 38 (C5), pp.C5-163-C5-165. 10.1051/jphyscol:1977521 . jpa00217170

\section{HAL Id: jpa-00217170 https://hal.science/jpa-00217170}

Submitted on 1 Jan 1977

HAL is a multi-disciplinary open access archive for the deposit and dissemination of scientific research documents, whether they are published or not. The documents may come from teaching and research institutions in France or abroad, or from public or private research centers.
L'archive ouverte pluridisciplinaire $\mathbf{H A L}$, est destinée au dépôt et à la diffusion de documents scientifiques de niveau recherche, publiés ou non, émanant des établissements d'enseignement et de recherche français ou étrangers, des laboratoires publics ou privés. 


\title{
MESURE DE LA FONCTION DE TRANSFERT ELECTRO-OPTIQUE D'UN SYSTĖME ÉLECTROCHIMIQUE $\left(^{*}\right)$
}

\author{
G. BLONDEAU, M. FROELICHER, C. GABRIELLI
}

A. HUGOT-LE GOFF et V. JOVANCICEVIC

Laboratoire de Physique des liquides et Electrochimie $\left({ }^{* *}\right)$

Université P. et M. Curie, 4, Place Jussieu, 75230 Paris Cedex 05, France

\begin{abstract}
Résumé. - On propose une nouvelle façon de mesurer la réponse optique en phase et en quadrature d'un interface électrochimique à une petite perturbation de potentiel. Le but est, au moyen d'un analyseur à fonction de transfert (AFT), d'étendre la technique de mesure aux basses fréquences (jusqu'à $10^{-4} \mathrm{~Hz}$ ). La comparaison avec les diagrammes d'impédance électrique montre que c'est dans ce domaine de fréquences que l'on peut mettre en évidence les processus de relaxation.
\end{abstract}

\begin{abstract}
A new way of measuring the in-phase and out-of-phase optical response of an electrochemical interface to a small perturbation of potential is proposed. The aim was, using a Transfer Function Analyzer (TFA), to develop the measurement technique at low frequencies (down to $10^{-4} \mathrm{~Hz}$ ). The comparison with electrical impedance diagrams proves in fact that 1elaxation pro cesses can be pointed out in this frequency range.
\end{abstract}

1. Introduction. - Une extension des mesures d'électroréflectance a été récemment proposée, basée sur la mesure conjointe des parties en phase et en quadrature du signal optique réfléchi $R$ au moyen d'une détection synchrone. L'exploitation des résultats peut ensuite se faire, soit par le tracé des parties réelle et imaginaire de $[\Delta R(\lambda) / \Delta V]_{\omega}$, pour une fréquence donnée $\omega$ de modulation du potentiel $V$ et à longueur d'onde variable $\lambda\left({ }^{1}\right)$, soit à partir du diagramme de la fonction de transfert électrooptique du système. Dans ce cas, la partie imaginaire de $[\Delta R(\omega) / \Delta V]_{\lambda}$ est portée en ordonnée et la partie réelle en abscisse pour une longueur d'onde déterminée, le diagramme étant paramétré en fonction de la fréquence de la perturbation sinusoïdale du potentiel $[1,2]$. Il nous a paru important d'étendre ce type de mesures vers les très basses fréquences car il a été montré [3] dans le cas des impédances électriques, que c'est dans ce domaine que se manifeste la relaxation des espèces présentes à l'interface. Si on suppose que les mêmes processus contrôlent le comportement électrique et le comportement optique de l'interface, on peut s'attendre à observer des fréquences propres de la fonction de transfert électrooptique dans le même domaine que celles de l'impédance électrique.

Pour effectuer des mesures à des fréquences inférieures à $1 \mathrm{~Hz}$ et pour réduire le bruit sans détruire l'information, il est nécessaire de traiter numérique-

(*) Ces résultats préliminaires ont été présentés au colloque à la place de la communication prévue. Ils font partie d'un texte destiné au 7th IVC \& 3rd ICSS, Vienne, 12-16 septembre 1977.

$\left.{ }^{* *}\right)$ Groupe de Recherche $n^{\circ} 4$, associé à l'Université $P$. et M. Curie.

(1) Wheeler D., Communication privée. ment le signal, donc d'utiliser une technique de corrélation. Un analyseur à fonction de transfert (AFT) permet d'obtenir directement $\Delta R / \Delta V$ dans un domaine de fréquences allant de $10^{4}$ à $10^{-4} \mathrm{~Hz}, \Delta V$ étant la modulation imposée du potentiel et $\Delta R$ la variation du pouvoir réflecteur de l'interface.

2. Résultats. - Ces résultats sont relatifs à l'oxydation anodique du titane, en solution sulfurique $1 \mathrm{~N}$ et pour une tension de $3 \mathrm{~V}$. Le titane est donc recouvert d'un film de $\mathrm{TiO}_{2}$ d'épaisseur $75 \AA$ [4]. La première figure présente un diagramme d'impédance électrique, obtenu par AFT. La boucle capacitive en haute fréquence correspond au film, la capacité correspondante diminue lorsque l'épaisseur de celui-ci croît,

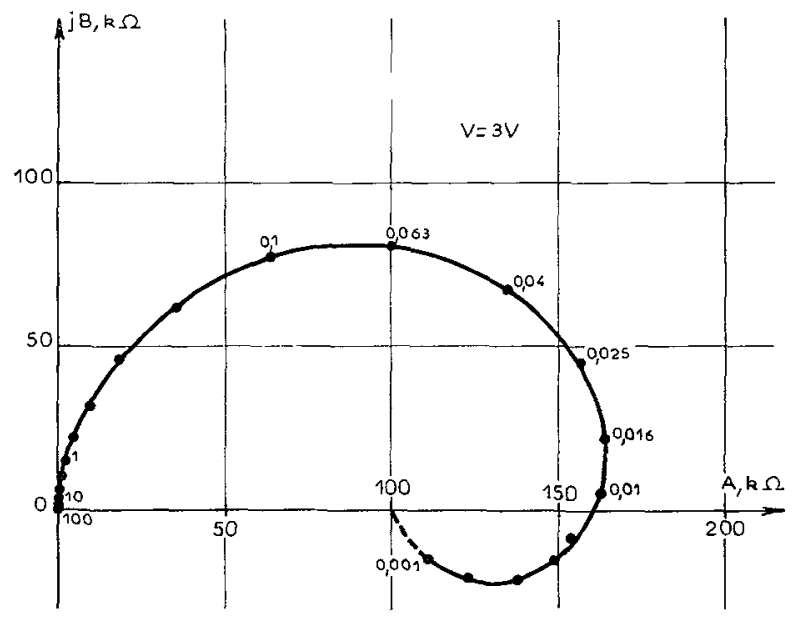

Fra. 1. - Diagramme d'impédance électrique paramètre en $\mathbf{H z}$ obtenu au cours de la polarisation anodique du titane en solution $\mathrm{H}_{2} \mathrm{SO}_{4} 1 \mathrm{~N}$. 
c'est-à-dire lorsque l'on se place à des tensions plus élevées. En basse fréquence on met en évidence une partie selfique qui va fortement se modifier si on fait varier la tension anodique. Sur la figure 2 , on a porté

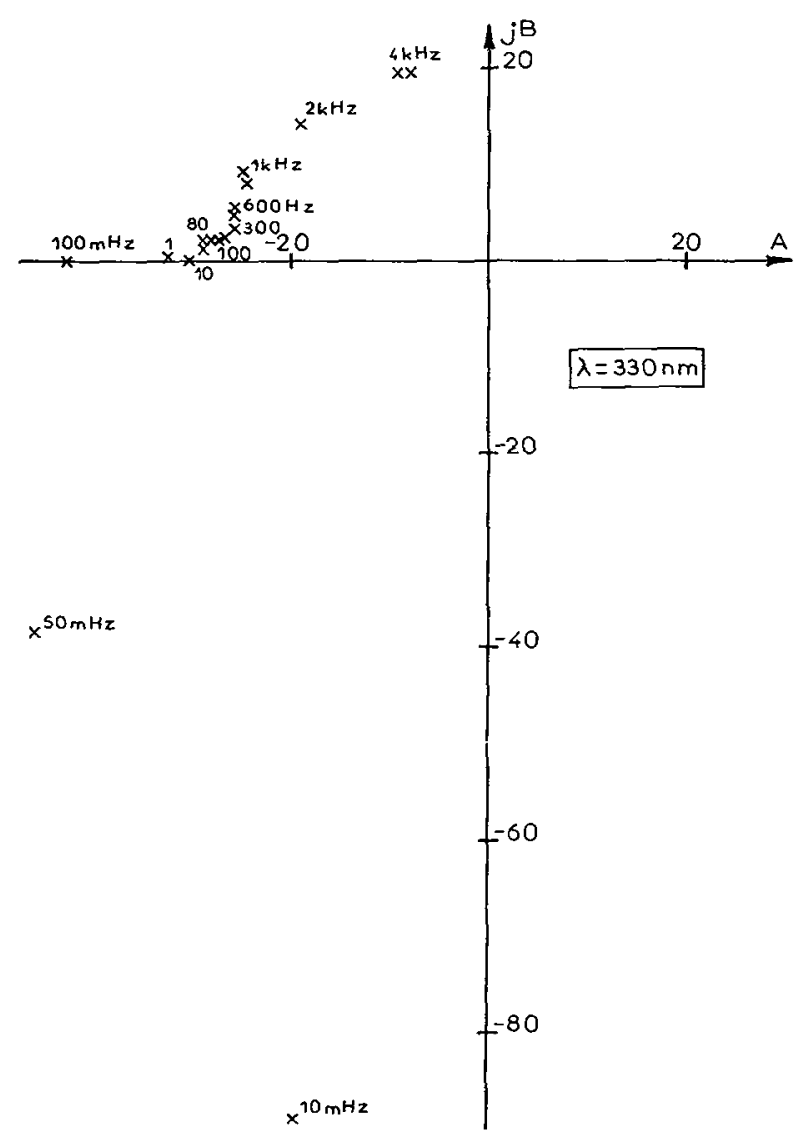

FIG. 2. - Fonction de transfert électro-optique obtenue dans les mêmes conditions que la figure 1 .

la fonction de transfert électrooptique du système, pour une longueur d'onde de $0,33 \mu \mathrm{m}: A$ correspond à la partie réelle de $\Delta R / \Delta V$ et $B$ à la partie imaginaire. Le bruit électrique lié en particulier au photomultiplicateur ne permet pas actuellement d'explorer la plus basse gamme de fréquence de modulation, on constate clairement que la réponse électrooptique de l'interface est modifiée dans la même gamme de fréquence de modulation que l'impédance électrique.

On constate aussi que dans la gamme $100 \mathrm{~Hz}-10 \mathrm{~Hz}$ environ ou sont effectuées les études d'électroréflectance classique, la réponse dépend très peu de la fréquence de modulation. On a porté sur la figure 3 , les

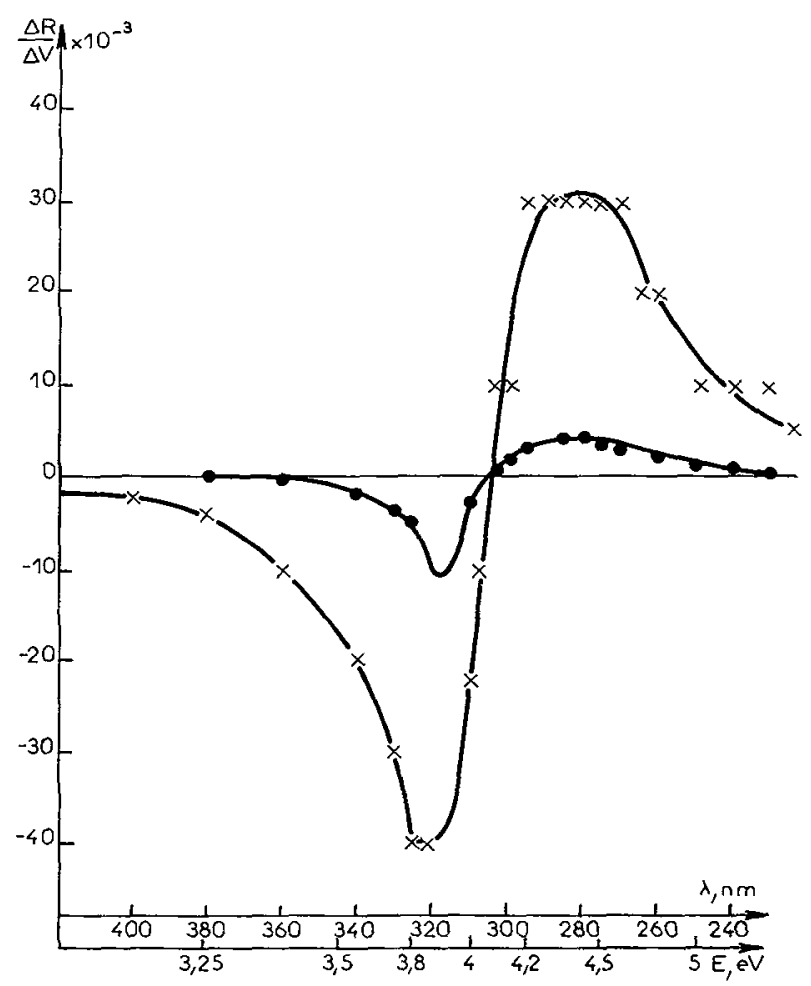

Fra. 3. - Partie réelle (croix) et imaginaire (points) de $\Delta R / \Delta V$ pour une fréquence de modulation de $30 \mathrm{~Hz} . \Delta V=100 \mathrm{mV}$.

parties réelle et imaginaire du diagramme d'électroréflectance obtenu avec une modulation de $30 \mathrm{~Hz}$ $(\Delta V=100 \mathrm{mV})$. Ces résultats sont, en ce qui concerne la partie réelle, parfaitement en accord avec les résultats de la littérature sur l'électroréflectance du rutile [5], ainsi qu'avec les résultats déjà publiés par Paatsch [6]. Cependant, on constate que même à cette fréquence de modulation, la partie en quadrature de la réponse est loin d'être négligeable. Il est assez difficile actuellement de dégager la signification exacte de la réponse en quadrature; il est à remarquer que le point singulier à $4,1 \mathrm{eV}$ associé au maximum de la constante diélectrique $\varepsilon_{2}$ de $\mathrm{TiO}_{2}(6,5)$, se retrouve dans cette réponse en quadrature.

Ce montage permet donc, d'une part d'obtenir des informations sur la structure de bande d'un film anodique en choisissant la gamme de fréquence de modulation la mieux adaptée, pour une étude en électroréflectance, d'autre part d'approcher les phénomènes à l'interface proprement dit par l'utilisation d'une modulation basse fréquence du potentiel.

\section{Bibliographie}

[1] Adzic, R., Cahan, B. et Yeager, E., J. Chem. Phys. 58 (1973) 1780.

[2] Dalbera, J. P., Hinnen, C. et Rousseau, A., J. Physique Colloq. 38 (1977) C4.

[3] Epelborn, I. et Keddam, M, J. Electrochem. Soc. 117 (1970) 1052.
[4] Blondeau, G., Froelicher, M., Froment, M. et HugotLe Gorf, A., Thin Solid Films 42 (1977) 147.

[5] Frova, A., Boddy, P. J. et Chen, Y. S., Phys. Rev. 157 (1967) 700.

[6] PaAtsch, W., Surf. Sci. 37 (1973) 59. 


\section{DISCUSSION}

YeAGER. - When an a. c. modulation is applied to an electrode with a highly irreversibly formed passivation layer, this situation may be quite complicated.

Can you elaborate on the factors contributing to the optical modulation, particularly at this very low frequencies $(<1 \mathrm{~Hz})$; and what types of informations you hope to obtain from these measurements at such low frequencies?

Froelicher. - Les études dans un large domaine de fréquences et particulièrement vers les TBF doivent être effectuées si l'on désire avoir des informations sur la cinétique comme l'ont montré les mesures d'impédances électrochimiques.

L'oxydation anodique du titane (comme tous les métaux valves) est un phénomène irréversible, aussi est-il nécessaire d'imposer une perturbation sinusoïdale de très faible amplitude - nous avons vérifié la linéarité du signal détecté pour une amplitude de la perturbation variant de 10 à $300 \mathrm{mV}$. Nous pensons donc que nous pouvons réeffectuer ces mesures uniquement dans le cas où l'on reste à un niveau de perturbation correspondant à la croissance d'une « mono-couche». 